\title{
A Novel Eigenface based Species Recognition System
}

\author{
T.A.S. Achala Perera \\ School of Engineering \\ Auckland University of Technology \\ New Zealand
}

\author{
John Collins \\ School of Engineering \\ Auckland University of Technology \\ New Zealand
}

\begin{abstract}
To develop a species recognition system for a resettable trap using novel species identification techniques.

Classical Eigenface based identification techniques are widely used in human faced detection domain. In this research Eigen faced based technique is used to identify the feral animals like Possums, cat and Weasels. When traditional Eigen faced technique is applied to detect these animal, detection rate is extremely poor (Possums 55\%, Cats 33\% and weasels 45\%), due to their orientation of the heads and fur patterns.

In this research, Eigenface based image recognition technique's detection rate was improved by adding different training sets to the system. Traditional Eigenface detection domain one training set is used, but it was discovered single training set was not adequate to detect small animal. This is because smaller animals like possums, cats and weasels tend to have different color group, different texture and hard to obtain face up images. Therefore it was decided to divide the training set into different sub groups. This sub training sets are used to train system and search for match. This method improved the detection rate up to $83 \%$ for possum, $50 \%$ for cats and $63 \%$ weasels.
\end{abstract}

\section{General Terms}

Euclidean distance, face recognition, animal detection

\section{INTRODUCTION}

In recent times machine vision based human detection systems have advanced rapidly. But there is very little research and development done in animal detection domain. Most of governmental and commercial agencies still used primitive technologies to detect animals.

An example, one of the most common practice is to identifying species, placing tracking tunnel using ink paper [1]. This method is currently used to identify, understand and study the species in a given area. At present there is an increasing demand for automated detection systems for animal monitoring, identifying and observing their behavior. These automated systems are cost effective, accurate and deployable in larger scale.

The main aim of this research is to investigate a vision based system to detect these feral animals. This smart system could replace traditional, footprint identifying expert based system. Final goal of this project is to use smart vision based technology to develop a resettable trap system, to target feral animals only. Also this vision based system can be used to study the local population of other species.

\section{CURRENT TECHNOLOGIES}

Currently there are few approaches to species recognition. The oldest technology is the use of an ink paper tunnel to identify the foot prints. Then most computer based systems use an artificial intelligence approach to train a system to identify the animal. The other approach is the use of image processing techniques to identify species.

\subsection{Identify Different Species by Footprints}

In this method animals firstly walk through an ink well and then walk over white paper. This method records the footprint information on the white paper (refer to Figure 1 below). Then this paper can be used to analyze the footprint information. There has been numerous research carried out to correlate the footprint information to actual species.

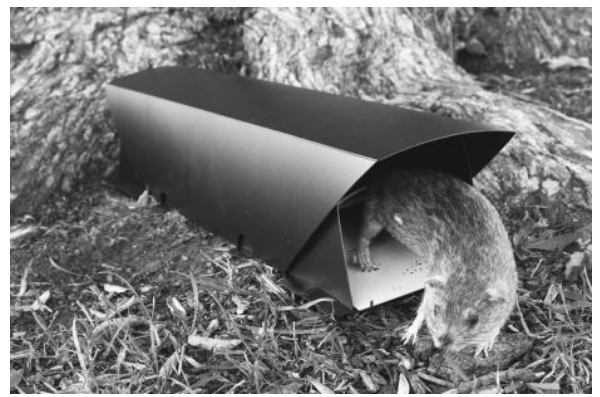

Figure 1 :Tracking Tunnel with tracking card[3]

Typically this type of research is carried out by scanning the tracking card, and using a pattern detection algorithm to extract the actual footprint from the background [3]. To attract the animals into the tunnels, lures in the tunnel are used [3, 4]. Both G. Yuan [4] and James' [3] independent research projects, use the technique of identifying footprint patterns by crosschecking using an existing footprint database. If any entry is matched with existing data, the animal can be identified.

The second method is measuring the distance between toe marks [5]. In this research, scanned footprints are extracted and then the distance between each artifact is measured. Using these measured data, the animal can be identified (refer to Figure 2 below)
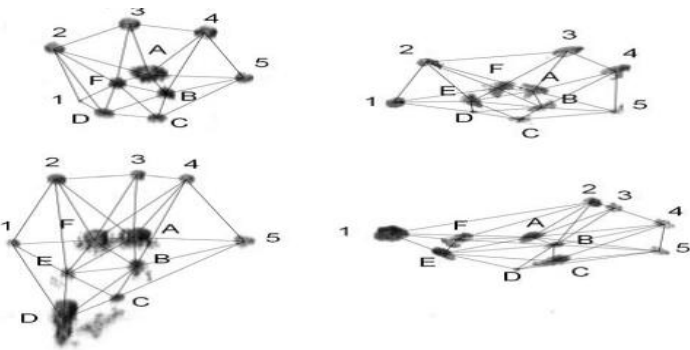

Figure 2: Measured Footprints [5]

\subsection{Artificial Intelligence Techniques}

Most computer based systems use an artificial intelligence approach. In general these systems required intensive computing power, hence more power. Most AI systems are based on human face detection. There is very little research carried on animal detection domain. This is due to human face detection being more predictable, with given face features. But with an animal there are different configurations, such as standing and sitting, and it is also a lot harder to extract animals 
from camouflaging environment background colors.

L. T. Antelo [6],used nonlinear modeling based artificial neural networks to identify different types of fish. They have used several techniques to identify fish species. The techniques are body shape, spatial color and weight estimation [6]. Once these combined details are extracted from the fish's image, this data is fed through artificial neural networks to identify the species [6].

These combinational approaches have better accuracy than any single identification technique. The accuracy of the system improves dramatically, when more than one feature is fed into the neural network system. Most neural network systems use image processing techniques to extract features from a given image and then these features are fed into the system to identify the specific pattern.

\subsection{Identifying Species by Image Processing Techniques}

Other research projects have used image processing techniques to identify animal and objects.

Object identification, by using image processing techniques is widely used in embedded platforms. This is because these systems have limited computational processing power. Naturally image processing tasks are power hungry. Therefore it is important to optimize system performance by introducing less computational power intensive image processing techniques. There are few research publications in the animal recognition domain using image processing techniques. All of these researches are carried out on larger animals, mostly due to ease of identification. Also most of the larger animals have unique skin features, sizes and shape which can be used to identify larger animal.

Pictorial structures have been used by D.Ramanan, Forsyth and Barnard to identify larger animals from video footage [7]. They have divided the animal into number of pictorial representations (rectangle structures). The configuration and orientation of these rectangle members of the animal can be used to identify the animal. Their research expanded into animal texture detection. They have developed a library of animal textures. By incorporating texture detection and pictorial representation, the accuracy of the system can be improved [7, 8].

T. Burghardt and J. Calic performed animal face detection and animal tracking using similar techniques as for human face detection [9, 10]. They used a combination of several techniques to identify lions' faces. Their detection strategy is based on Viola-Jones detector techniques [11]. Also Haar-like features [12] and AdaBoost are integrated into their system for smooth and accurate tracking $[9,10]$. These human face detection techniques can be used in the animal recognition domain reasonably accurately. According to their research, the detection rate for larger animals is high (refer to Figure 3 below).

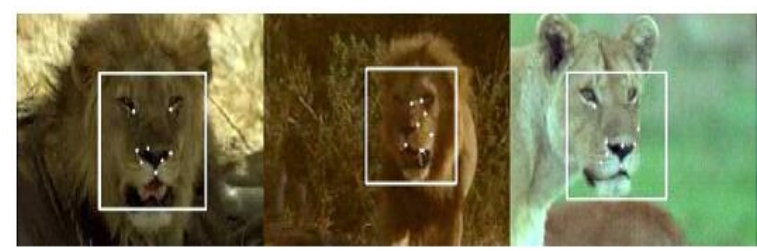

Figure 3: Feature Point Stipulated in the center of the detected region[9]
W. Zhang, J. Sun and X. Tang used both texture and features to detect larger animals like tigers, panda, fox, cat and cheetah [2]. Their system uses Histogram of Oriented Gradients (HOG) [13], to capture the shape and texture features on the animal's head. Once fur texture and features are captured, Brute force detection and Deformable detection algorithms are used to effectively exploit the shape feature and texture features concurrently [2].

The above research [2] shows a promising results for animal recognition. The combined approach of fur texture and face detection have the best animal identification rate. Currently these types of combined approaches are only attempted on larger mammals such as lion, tigers, cats, etc. The use of the combinational approach for small animal detection and identification in real-time is a novel approach.

\section{EIGENFACES}

Eigenfaces is set of eigenvectors, which are used to detect human faces [14-16]. In this application eigenvectors are used to detect cats, possums and weasels.

The Eigenfaces algorithmic computation can be broken down to few steps $[14,16,17]$.

Step 1: Obtaining the training set images $I_{1}, I_{2}, \ldots \ldots ., I_{M}$

Step 2: Convert each image $I_{i}$ into a vector $\Gamma_{i}(N \mathrm{x} N$ image into $N^{2} \mathrm{x} l$ vector)

Step 3: Calculate the average face vector $\Psi$ :

Step 4: $\quad$ Subtract the average face from $\Gamma_{i}$ to get $\Phi_{i}$ :

$$
\Phi_{i}=\Gamma_{i}-\Psi
$$

Step 5: Calculate the covariance matrix C:

$$
\left(\mathrm{N}^{2} \mathrm{xN}^{2}\right. \text { matrix) }
$$

Where $\left(\mathrm{N}^{2} \mathrm{xM}\right.$ matrix $)$

Step 6: Calculate the eigenvectors $u_{i}$ of [16]

These are same as $M$ best eigenvectors from best eigenvalues.

Once best eigenvector from training set is computed, unknown images can be feed through the system for face recognition. Before the recognition input image need to be normalized and demeaned. This processed can be split into four steps for a given unknown image $\Gamma[14,15]$.

Step 1: Calculate $\Phi=\Gamma-\Psi$

Step 2: Calculate

Step 3: Calculate Euclidean distance [16]

Step 4: If then $\Gamma$ is a face

In typical application, Euclidean distance is calculated. Then this distance is compared against a known threshold value. If the Euclidean value is less than the threshold, the input unknown image is one of the training set images. Otherwise it is not.

\section{SUB TRAINING SET BASED EIGENFACES}

Sub training set based system is a technique that uses different training sets for same animals. These training sets are optimized according to animals' color and camera pose angle. 


\subsection{Why Sub Training Set based Technique (Problems)}

In animal detection domain the underlying problem for Eigenfaces technique is all the animals have different texture patterns, color variation and face features. In some cases same animal have color variation. As an example, possums have two different colors gray and dark brown colored possum (refer to Figure 4).The problem with this color variation is when these images are fed into training set as a possum, accuracy of the system deteriorates rapidly.

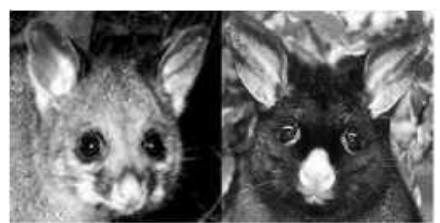

Figure 4: Different colored possums

The second problem is finding images of animal in correct orientation. Most animal tend to have different poses. In typical Eigenface application all the faces need to be oriented in correct format (looking forward). Training set with incorrect oriented images deteriorates the accuracy of the system $[14,17]$.

Due to above reasons, detection rate of the Eigenface technique is very poor. Therefore to overcome this problems sub category training set based Eigenfaces technique was developed. In this technique all the likely subjects, grouped into one category. As an example all the dark colored possum in one category and light colored possum in another category. In order to improves the system accuracy further, these categories are broken down into further sub groups like light colored possum looking left, light colored possum looking right and so on (refer to Figure 5). Then each of these categories used as a separate training set.

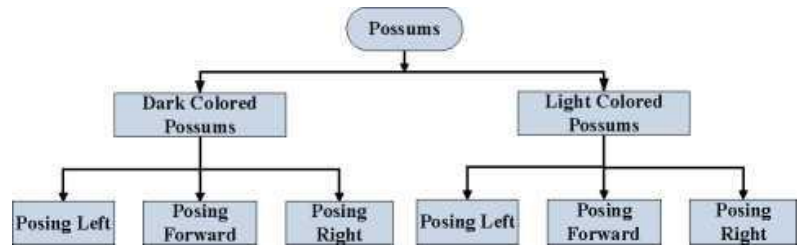

Figure 5: Sub categorizing the animals

Animal like cats and weasels are hard to grope into fur color based groups. These animals have different colors and patterns on their fur. It was found the best way to categorize these animals, by their pose. As an example cats and weasels are categorized by posing left, posing forward and posing right (refer to Figure 6)

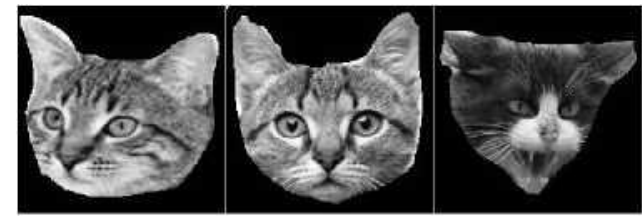

Figure 6: Cat faces posing different directions

\subsection{Training Set Selection}

During the initial stages of experiments, animal images with different backgrounds were used. Animal images with random backgrounds produce strong Eigenvectors primarily related to the background information. These images further reduce the detection rate.

Therefore all the training set images had been preprocessed. The background has been replaced with a uniform black color (refer to Figure 7). Now all the Eigenvalues have very strong correlation with the actual animal face rather than the background.

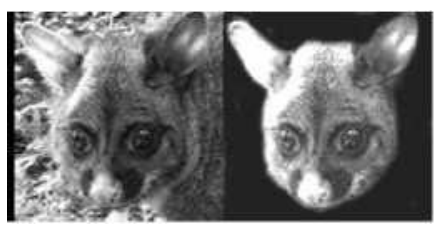

Figure 7: Possum Image before and after prepossessed

\subsection{Computational Process}

The computational steps are mostly followed as Eiengface detection. The different is; there are multiple sets of training data sets need to be formatted. Then these data sets are used to generate the Eigenfaces for given image. These algorithms can be express as follow.

Obtaining the training sets 'Ii' for dark colored possums posing left (PDL), forward (PDF), right (PDR) and light colored possum posing left (PLL), forward (PLF), and right (PLR)

Ii(PDL), Ii(PDF), Ii(PDR),Ii(PLL), Ii(PLF), Ii(PLR)

Convert training sets into vectors:

Гi(PDL), Гi(PDF),Гi(PDR), Гi(PLL), Гi(PLF),Гi(PLR)

Calculate the average face for each training set:

(PDL),(PDF),(PDR),(PLL),(PLF),(PLR)

Calculate the average face for each training set:

Фi(PDL), Фi(PDF),Фi(PDR),Фi(PLL), Фi(PLF),Фi(PLR)

Calculate the covariance matrixes for each training set:

C(PDL), C(PDF), C(PDR), C(PLL), C(PLF), C(PLR)

Calculate the Eigenvectors uifor each training set: ui(PDL), ui(PDF),ui(PDR),ui(PLL),ui(PLF),ui(PLR)

Select and keep best Eigenvectors K from AAT matrixes of each training set:

\section{K(PDL),K(PDF),K(PDR),K(PLL),K(PLF),K(PLR)}

When a given unknown image is fed into the system; the system reconstructs an Eigenface from each training set. Then the Euclidean distance ed for each reconstructed Eigenface from different training set is calculated.

ed(PDL), ed(PDF), ed(PDR), ed(PLL), ed(PLF), ed(PLR)

Then best match is selected from the calculated Euclidean distances. It was found this selection process is more accurate than typical Eigenface detection.

\section{RESULTS}

\subsection{Practical Scenario}

Compare with typical Eigenfaces, sub training set based Eigenfaces technique has significant improvement on detection rate (refer to Table 1 and Figure 8). 
Table 1: Animal Detection Rates

\begin{tabular}{|c|c|c|}
\hline Animals & $\begin{array}{c}\text { Typical } \\
\text { Eigenfaces }\end{array}$ & $\begin{array}{c}\text { Sub-Training } \\
\text { Set Based } \\
\text { Eigenfaces }\end{array}$ \\
Possums & $55 \%$ & $83 \%$ \\
\hline Cats & $33 \%$ & $50 \%$ \\
\hline Weasels & $45 \%$ & $64 \%$ \\
\hline
\end{tabular}

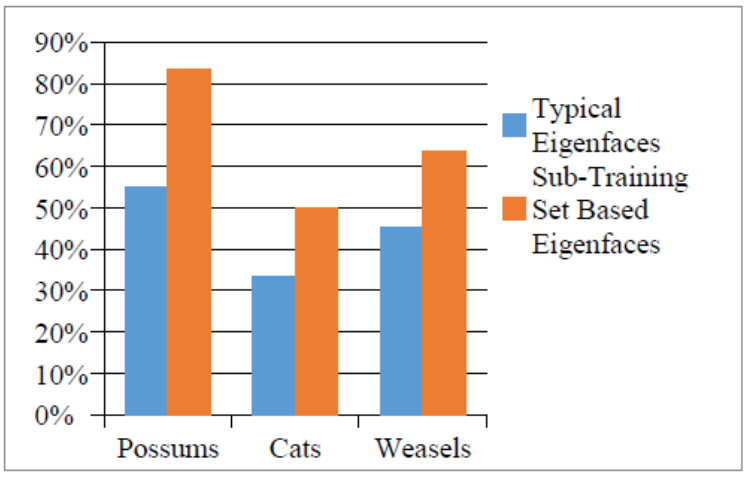

Figure 8: Animal Detection Rate

Possum detection has the highest accuracy. This is because once all the sub training sets were allocated, actual difference on possums is minimal. On the other hand, cats have huge variation. They have different facial fur patterns and colors. Hence they have lowest detection rate.

Even though weasels have different facial colors and patterns, there head size and main features like nose and ears are distinct. Therefore they have good detection rate with sub training set based Eigenfaces. In this case sub training set based system works better compare with Eigenface technique. This is because sub training set can train the system to detect deferent poses (looking left or right) accurately.

\subsection{Ideal Scenario}

Test images without background have been fed into the system to check the performance in ideal case. In this case there is significant improvement in detection rate. This is because all the Eigen values have significant representation of the animal face rather than the background information.

Table 2: Animal Detection Rates

\begin{tabular}{|c|c|c|}
\hline \multicolumn{3}{|c|}{ Test Images without Background } \\
\hline Animals & $\begin{array}{c}\text { Typical } \\
\text { Eigenfaces }\end{array}$ & $\begin{array}{c}\text { Sub-Training Set } \\
\text { Based Eigenfaces }\end{array}$ \\
\hline Possums & $65 \%$ & $93 \%$ \\
\hline Cats & $52 \%$ & $67 \%$ \\
\hline Weasels & $64 \%$ & $82 \%$ \\
\hline
\end{tabular}

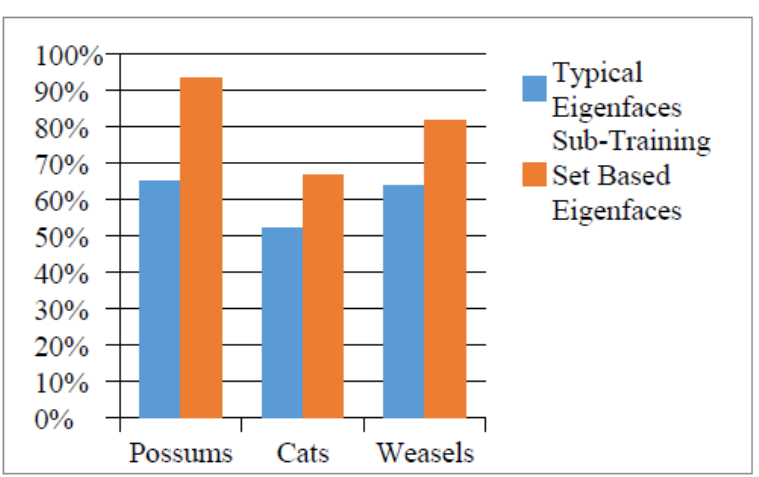

Figure 9: Animal Detection Rate

\subsection{Optimal Training Set Size}

One of the main dictating factors for accuracy is training set size. It was found sub training set based Eigenfaces requires a minimal number of training images to archive high detection rate. According to the experiment below with minimum of 5 training images are required (refer to Figure 10 ). On the other hand Eigenfaces technique requires, at least 18 or more training images to achieve same detection rate as sub-training set based method.

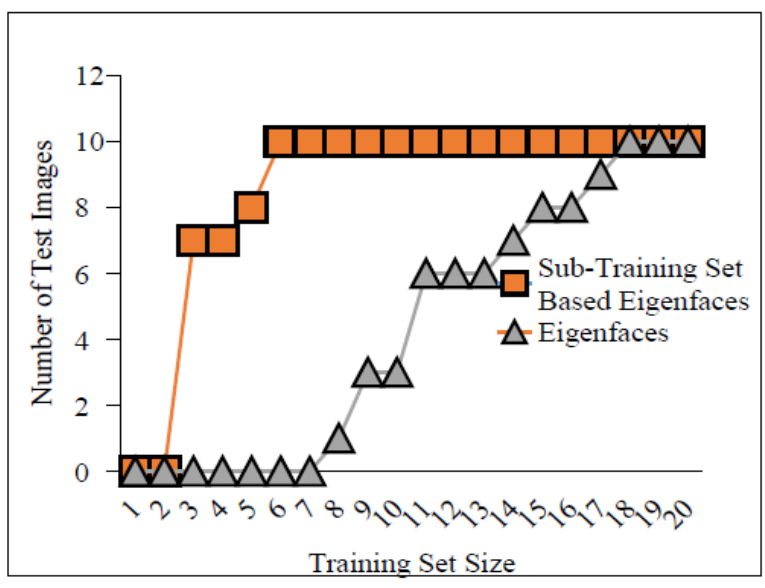

Figure 10: Detection Rate Vs Sample Size

\section{CONCLUSION}

Overall there is considerable gain in this method. On an average there is a $31 \%$ improvement on detection rate. The main advantage of the Eigenface based technique is most of computationally intensive tasks can be done prior detection time. During the detection time there is minimal number of steps of computations to follow. This technique is ideal to implement in low resources based embedded system application.

The next stage of this project is to develop a smart trap system using this novel species recognition approach. The final system will comprise with Advanced RISC Machines (ARM) based processor with a camera system to detect wild rodents. This type of system can be used to control the pest population buy smart trapping or can be used to study the wild animal population. The developed system will be far superior to existing elementary systems. This proposed system will remove the requirement of specialized trained expert to detect animal either by their foot print pattern or fur samples.

Also sub training set based technique can be introduced into human detection domain to improve the accuracy. 


\section{REFERENCES}

[1] D. W. Craig Gillies, "A short guide for identifying footprints on tracking tunnel papers," vol. OLDDM-63018 1

[2] Z. Weiwei, S. Jian, and T. Xiaoou, "From Tiger to Panda: Animal Head Detection," Image Processing, IEEE Transactions on, vol. 20, pp. 1696-1708, 2011.

[3] N. H. JAMES C. RUSSELL, REINHARD KLETTE, AND BODO ROSENHAHN, "Automatic track recognition of footprints for identifying cryptic species," the Ecological Society of America, vol. 90, pp. 2007 2013,2009 .

[4] N. H. Guannan Yuan, James Russell, Reinhard Klette, and Bodo Rosenhahn, "Understanding Tracks of Different Species of Rats," The University of Auckland, Auckland 187, 2006.

[5] R. G.-G. Alexandre R. T. Palma, "Morphometric identification of small mammal footprints from ink tracking tunnels in the Brazilian Cerrado," Revista Brasileira de Zoologia, vol. 24, pp. 333 - 343, 2007.

[6] L. T. Antelo, T. Ordonez, I. Minino, J. Gracia, E. Ribes, J. Hervas, S. Simon, and A. A. Alonso, "A vision-based system for on-board identification and estimation of discarded bio-mass: A tool for contributing to marine resources sustainability," in OCEANS, 2011 IEEE Spain, pp. 1-8.

[7] Ramanan D., Forsyth D. A., and Barnard K., "Building models of animals from video," Pattern Analysis and Machine Intelligence, IEEE Transactions on, vol. 28, pp. 1319-1334, 2006.

[8] K. J. Dana, S. K. Nayar, B. van Ginneken, and J. J. Koenderink, "Reflectance and texture of real-world surfaces," in Computer Vision and Pattern Recognition, 1997. Proceedings., 1997 IEEE Computer Society Conference on, 1997, pp. 151-157.

[9] T. Burghardt and J. Calic, "Real-time Face Detection and Tracking of Animals," in Neural Network Applications in
Electrical Engineering, 2006. NEUREL 2006. 8th Seminar on, 2006, pp. 27-32.

[10] T. Burghardt and J. Calic, "Analysing animal behaviour in wildlife videos using face detection and tracking," Vision, Image and Signal Processing, IEE Proceedings -, vol. 153, pp. 305-312, 2006

[11] P. Viola and M. J. Jones, "Robust Real-Time Face Detection," Int. J. Comput. Vision, vol. 57, pp. 137-154, 2004.

[12] R. Lienhart and J. Maydt, "An extended set of Haar-like features for rapid object detection," in Image Processing. 2002. Proceedings. 2002 International Conference on, 2002, pp. I-900-I-903 vol.1.

[13] P. Viola and M. Jones, "Robust real-time face detection," in Computer Vision, 2001. ICCV 2001. Proceedings. Eighth IEEE International Conference on, 2001, pp 747-747.

[14] M. A. Turk and A. P. Pentland, "Face recognition using eigenfaces," in Computer Vision and Pattern Recognition, 1991. Proceedings CVPR '91., IEEE Computer Society Conference on, 1991, pp. 586-591.

[15] M. Smiatacz, "Eigenfaces, Fisherfaces, Laplacianfaces, Marginfaces - How to Face the Face Verification Task," in Proceedings of the 8th International Conference on Computer Recognition Systems CORES 2013. vol. 226, R. Burduk, K. Jackowski, M. Kurzynski, M. Wozniak, and A. Zolnierek, Eds., ed: Springer International Publishing, 2013, pp. 187-196.

[16] M. Turk and A. Pentland, "Eigenfaces for Recognition," Cognitive Neuroscience, Journal of, vol. 3, pp. 71-86, 1991.

[17] P. N. Belhumeur, J. P. Hespanha, and D. Kriegman, "Eigenfaces vs. Fisherfaces: recognition using class specific linear projection," Pattern Analysis and Machine Intelligence, IEEE Transactions on, vol. 19, pp. 711-720, 1997. 